\title{
Corela
}

Cognition, représentation, langage

6-1 | 2008

Vol. $6, n^{\circ} 1$

\section{Prépositions et langage chez de jeunes sourds profonds oralistes}

\author{
Laurence Vincent-Durroux
}

\section{(2) OpenEdition}

Journals

Édition électronique

URL : http://journals.openedition.org/corela/259

DOI : $10.4000 /$ corela.259

ISSN : $1638-573 \mathrm{X}$

Éditeur

Cercle linguistique du Centre et de l'Ouest - CerLICO

Référence électronique

Laurence Vincent-Durroux, «Prépositions et langage chez de jeunes sourds profonds oralistes », Corela [En ligne], 6-1 | 2008, mis en ligne le 24 juin 2008, consulté le 04 mai 2019. URL : http:// journals.openedition.org/corela/259; DOI : 10.4000/corela.259

Ce document a été généré automatiquement le 4 mai 2019.

\section{(c) (i) (2)(2)}

Corela - cognition, représentation, langage est mis à disposition selon les termes de la licence Creative Commons Attribution - Pas d'Utilisation Commerciale - Partage dans les Mêmes Conditions 4.0 International. 


\title{
Prépositions et langage chez de jeunes sourds profonds oralistes
}

\author{
Laurence Vincent-Durroux
}

1 En limitant considérablement l'audition ${ }^{1}$, la surdité profonde prélinguistique limite fortement l'accès à l'environnement linguistique oral dont bénéficient les enfants entendants. L'ouïe est également une voie privilégiée pour la construction des représentations de temps et d'espace, dimensions cognitives impliquées dans le langage. Or, "le monde est sans profondeur pour l'enfant sourd" (Lafon, 1985 : 19), du moins tant qu'il ne bénéficie pas de compensation prothétique très efficace ${ }^{2}$. La surdité peut donc avoir des conséquences qui se manifestent dans les productions linguistiques des sourds.

2 Les travaux de recherche consacrés aux sourds ont d'abord fait apparaître les écarts entre la production de ces derniers et la langue standard, et ont porté presque exclusivement sur l'écrit, pour des raisons d'intelligibilité (pour une synthèse: Vincent-Durroux, 1992, chapitre 2). Une hypothèse formulée par Charrow (1976) a ouvert des perspectives nouvelles: sur la base des régularités observées dans leurs productions, Charrow a proposé que la langue (non signée) des sourds américains soient une langue à part entière (Deaf English); cette hypothèse a pu être exploitée dans des travaux tels que ceux de Lacerte (1988) et Vincent-Durroux (1990 \& 1992), menés sur le québécois, le français et l'anglais. Enfin, avec le développement de l'implant cochléaire dans les années 1990, des études ont été faites pour évaluer le bénéfice des patients en termes de perception et d'intelligibilité (Miyamoto et al., 1999), en croisant les données avec le mode de communication, l'âge et la durée d'implantation (O'Donoghue, 1999 ; Geers et al., 2000). D'autres études ont été consacrées à la progression lexicale (Ouellet et al., 2001) ou à la progression syntaxique (Vieu et al., 1998). Notre approche présente l'originalité de s'appuyer sur des données orales recueillies dans deux langues différentes; leur analyse syntaxico-sémantique et la mise en évidence de points communs peuvent permettre de tester des hypothèses sur les spécificités du langage des sourds profonds. 


\section{Présentation du corpus d'étude}

3 Nous disposons de deux corpus oraux, recueillis en 1988 et en 2006 auprès de sujets dont les parents ont fait le choix de l'oralisme ${ }^{3}$ : il s'agit pour les enfants d'utiliser la langue de leur communauté (l'anglais ou le français) ; le recours à une langue signée est exclu.

4 En vue de comparaisons éventuelles, le protocole d'enregistrement a été conservé d'un corpus à l'autre. Le protocole prévoit des enregistrements sur support audio, en temps limité, avec une batterie de cinq exercices destinés à favoriser la production de plusieurs types de discours. Il s'agit de discours descriptifs (avec support : description d'une image ; sans support: description de la maison du sujet), de discours narratifs (avec support: séquence d'images; sans support: raconter une fête d'anniversaire) et de discours argumentatifs (réagir à une interdiction prononcée par une personne en situation de responsabilité à l'égard du sujet).

5 Les sujets retenus ont une surdité profonde, congénitale, bilatérale et prélinguistique. Il s'agit de 4 anglophones et 4 francophones en 1988 et de 6 anglophones et 6 francophones en 2006. Les sujets étaient âgés de 12 à 15 ans en 1988, et soit de 10 à 12 ans, soit de 13 à 15 ans en 2006 (Tableau 1).

Tableau 1 : Caractéristiques des sujets enregistrés

\begin{tabular}{|l|l|l|l|}
\hline Corpus & Age des sujets & Langue & Nombre de sujets \\
\hline 1988 & $12-15$ ans & $\begin{array}{l}\text { anglais } \\
\text { français }\end{array}$ & 4 \\
\hline 2006 & $\begin{array}{l}10-12 \text { ans } \\
13-15 \text { ans }\end{array}$ & $\begin{array}{l}\text { anglais } \\
\text { français } \\
\text { anglais } \\
\text { français }\end{array}$ & 3 \\
\hline
\end{tabular}

6 L'âge a été défini pour le premier corpus sur la base d'études indiquant l'absence de progression notable dans les années ultérieures à la pré-adolescence (Bochner et Albertini, 1988). Les différences d'âge et de nombre de sujets entre les deux corpus sont justifiées par les changements intervenus entre ces deux périodes et leurs répercussions probables sur la qualité des productions orales. En effet, en 1988, les sourds profonds oralistes étaient pourvus de prothèses auditives classiques leur procurant un gain auditif faible à moyen. En revanche, les sujets enregistrés en 2006 bénéficiaient tous d'un implant cochléaire identique depuis au moins 7 ans. L'implant cochléaire est un dispositif implanté par voie chirurgicale sous les cheveux et dans l'oreille ; il comporte un système électronique piloté de l'extérieur par un émetteur caché derrière l'oreille. Ce dispositif peut améliorer considérablement la perception auditive, qui peut être évaluée comme étant moyenne à bonne. La question suivante se pose alors : le gain auditif obtenu dans ces conditions permet-il aux sujets sourds de produire une langue orale spontanée libérée des caractéristiques décrites chez les sujets pourvus de prothèses classiques? 
7 Dans l'ensemble, 4200 énoncés ont été enregistrés, transcrits et analysés (en 1988: 2312 énoncés, soit 18817 mots ; en 2006: 1896 énoncés, soit 15088 mots). Des locuteurs témoins entendants ont été enregistrés avec le même protocole, en anglais et en français.

8 L'étude présentée ici est centrée sur l'emploi des prépositions dans les corpus. Cette analyse peut en effet nous renseigner sur certains aspects du langage chez les sourds dans la mesure où les prépositions sont notamment liées à la prédication, à l'espace et au temps. En effet, les prépositions sont des relateurs entre des notions dont l'une d'elles au moins est argumentale (Franckel et Paillard, 2007); l'emploi des prépositions par les sourds peut donc faire apparaître des spécificités dans les opérations de prédication, opérations centrales dans le langage. Par ailleurs, dans les deux langues sur lesquelles portent nos observations, de très nombreuses prépositions trouvent leur origine dans les domaines de l'espace et du temps : "sur une liste de 60 prépositions de l'anglais actuel, seulement 7 ne remontent pas à une indication de relation dans l'espace" (Groussier, $1997: 222)$.

9 Les exercices $\mathrm{du}$ protocole sollicitent des productions sous-tendues par les représentations spatio-temporelles des sujets, représentations pouvant notamment être figurées par des prépositions. Les productions comportent en effet de nombreuses prépositions, mais si, chez les locuteurs témoins, aucune d'elles ne s'écarte de la langue standard, ce n'est pas le cas dans les productions des sourds, et ce, dans les deux corpus.

\section{Eléments quantitatifs}

Les deux corpus comportent des énoncés jugés agrammaticaux en relation avec les prépositions. L'analyse quantitative révèle 98 cas de prépositions problématiques en 1988 et 59 cas en 2006. Une approche formelle de ces différents cas permet de faire une répartition en trois catégories (Tableau 2):

- la préposition est superflue dans le contexte :

(1) le mur elle avait avec un miroir [MJL4];

- la préposition produite n'est pas adaptée au contexte :

(2) après il va dans la caissière [MJL17];

- la préposition est absente là où elle serait attendue en langue standard :

(3) ils rentrent leur lit [MR41].

Tableau 2 : Répartition des prépositions problématiques (nombre de cas et proportions)

\begin{tabular}{|l|l|l|}
\hline Corpus & 1988 & 2006 \\
\hline Prépositions superflues & $12=12,2 \%$ & $10=17 \%$ \\
\hline Prépositions inadaptées & $37=37,8 \%$ & $26=44 \%$ \\
\hline Prépositions absentes & $49=50 \%$ & $23=39 \%$ \\
\hline Total (prépositions problématiques) & $98=100 \%$ & $59=100 \%$ \\
\hline
\end{tabular}

11 Nous pouvons certes observer une diminution globale du nombre de prépositions problématiques pour des énoncés en quantités comparables dans les deux corpus. 
Proportionnellement, les prépositions superflues ou inadaptées au contexte augmentent, et les prépositions absentes diminuent. En 2006, malgré des conditions d'audition meilleures, le taux de prépositions absentes reste toutefois élevé, ce qui nous conduit à prendre en compte cette catégorie de façon détaillée (paragraphe 4).

\section{Linéarité dans l'énoncé et prédication}

L'analyse des énoncés qui comportent des prépositions superflues ou des prépositions inadaptées au contexte dans lequel elles se trouvent, fait bien souvent apparaitre des problèmes de linéarité syntaxique à des degrés variables.

\subsection{Transitivité}

La présence d'une préposition superflue peut révéler une structuration prédicative erronée. Cela peut concerner la transitivité dans la relation prédicative, comme dans l'énoncé (4) :

(4) prendre le train avec le train [MR49]

Cet énoncé présente une relation prédicative initiale transitive ; le déterminant "le" qui suit le verbe immédiatement en est l'indice $:<X$, PRENDRE, TRAIN $>\rightarrow X$ prendre le [train]. Toutefois, avant de produire le complément, l'énonciateur produit la préposition "avec", inadaptée au verbe "prendre" mais compatible avec d'autres verbes comme "voyager" ; le verbe n'est toutefois pas modifié par un phénomène de reprise, ce qui donne l'impression d'une confusion entre deux structures (prendre le train / voyager avec le train), entrainant le décumul de deux relations, l'une étant locative (prendre le train), l'autre étant instrumentale (voyager avec le train).

\subsection{Rôles thématiques}

16 La mise en place des rôles thématiques peut être exprimée par le choix des prépositions. Nous observons le verbe "partager" dans les corpus, dans son emploi où il y a deux bénéficiaires dans le partage (celui qui partage et celui avec lequel il partage). L'énoncé retenu ici se situe dans un discours argumentatif : le sujet donne un argument en vue de faire changer ses parents d'avis afin qu'ils l'autorisent à partir en Italie. Son argument consiste à partager la dépense ("l'argent") avec eux :

(5) je partage un peu l'argent pour mes parents [MS73]

17 Les référents de "je" et de "mes parents" ont en fait le même rôle thématique vis-à-vis du prédicat ; "pour" est inadapté car il est incohérent avec le contexte.

\subsection{Copule et séparabilité}

Les corpus comportent plusieurs occurrences de localisations spatiales ou temporelles dont l'association à des prépositions provoque des agrammaticalités. Ces occurrences de localisation peuvent être ramenées à des schémas copulatifs à valeur locative impliquant $\mathrm{BE}$ : 
(6) and I've got a very long garden with a tree in half way through [AX1]

$\rightarrow$ TREE BE IN GARDEN (I've got a very long garden with a tree in) + TREE BE Ø HALF

WAY THROUGH (I've got a very long garden with a tree half way through) ;

(7) and I can see a cat on the end of his bed

$\rightarrow$ CAT BE ON HIS BED (I can see a cat on his bed) + CAT BE [AT] THE END OF HIS BED

(I can see a cat at the end of his bed) ;

(8) and it was very hot because it is in June 19th

$\rightarrow$ BIRTHDAY BE IN JUNE (it is in June) + BIRTHDAY BE [ON] JUNE $19^{\text {th }}$ (it is on June $\left.19^{\text {th }}\right)$.

On remarque que pour chaque occurrence, le déroulement linéaire de l'énoncé permet d'augmenter le degré de précision du lieu (énoncés 6 et 7) ou du temps (énoncé 8). A chaque fois, deux éléments copulatifs devraient être impliqués. Or la forme observée ne présente qu'un seul $\mathrm{BE}$, avec une préposition unique qui n'est pas adaptée aux deux schémas copulatifs sous-jacents. Ce type d'énoncés montre que pour nos sujets, il pourrait y avoir une séparabilité de la copule et de son élément de droite, séparabilité dont témoigne l'emploi problématique de la préposition.

\subsection{Confusion de statuts et rupture de la linéarité}

L'emploi des prépositions peut aller de pair avec un rôle sémantique ne permettant plus au groupe prépositionnel d'occuper certaines fonctions syntaxiques dans la relation prédicative. Par exemple, l'emploi de "pour" suivi d'un groupe nominal en début d'énoncé peut conférer au groupe prépositionnel le rôle sémantique de thème, et empêcher ce groupe nominal (exemple 9) d'être complément d'objet direct (COD). La suppression de "pour" donne la possibilité au groupe nominal d'être COD (exemple 9') :

(9) Pour mon dernier anniversaire, j'ai fait du ski nautique.

(9') Mon dernier anniversaire, je l'ai passé à Londres.

21 L'énoncé (10) peut être jugé agrammatical pour la raison ci-dessus, d'autant plus qu'aucune pause n'intervient avant "parce que" :

(10) alors pour mon dernier anniversaire je l'ai passé à parce que moi j'étais parti en vacances à Colmar [TM9]

Le choix de commencer ainsi l'énoncé bloque la reprise de "pour mon dernier anniversaire" en tant que COD, y compris sous forme pronominale. La mise en place d'un thème est bien distincte d'une structure avec dislocation à gauche dans laquelle l'élément déplacé vers la gauche peut être COD.

Ainsi l'emploi erroné de la préposition dans l'énoncé manifeste la confusion possible chez les sujets entre des statuts sémantiques et syntaxiques incompatibles, aboutissant à une rupture de la linéarité de l'énoncé, rupture pouvant être déstabilisante pour l'auditeur. Les occurrences analysées ci-dessus montrent qu'il existe des spécificités dans l'emploi des prépositions chez les sourds, spécificités affectant la relation prédicative à différents niveaux.

\section{Temps et espace}

L'audition est un vecteur privilégié pour la construction des représentations spatiotemporelles (Jimenez, 1997). En analysant des énoncés jugés problématiques en relation avec la préposition, il devrait être possible d'établir s'il y a une appréhension, et partant, 
une représentation du temps et de l'espace qui soit propre aux sourds ( $c f$. la fin du paragraphe 1 pour les rapports entre préposition, temps et espace).

Pour cela, nous nous intéressons plus particulièrement aux prépositions absentes. Il y en a 72 cas dans les corpus ; plusieurs hypothèses permettent d'en rendre compte.

\subsection{Hypothèse phonologique}

La première hypothèse est phonologique. En relation avec la surdité en effet, on peut supposer que la perception plus ou moins bonne des prépositions peut avoir une influence sur leur production. Si on prend l'exemple de l'anglais, plusieurs prépositions monosyllabiques très courantes sont le plus souvent inaccentuées et associées à des voyelles réduites ; elles sont donc difficiles à percevoir, surtout si leur contexte immédiat comporte la même consonne, comme dans les exemples (11) à (13) :

(11) I'm looking at Tom.

(12) I'm going to Toronto.

(13) It's a picture of Venice.

Lorsque la langue est en construction, la perception joue un rôle important. Dans le contexte de l'acquisition d'une langue étrangère par exemple, la mauvaise perception de certains éléments de la langue (notamment du fait de syllabes inaccentuées et de réductions vocaliques) entraîne des difficultés de compréhension mais conduit également à la construction par l'apprenant de règles erronées, et donc d'erreurs en production écrite ou orale (Huart, 2002). Pour les sourds, la perception de la langue orale est parfois lacunaire, ce qui pourrait expliquer certaines productions telles que les énoncés (14) à (16), pour lesquels nous indiquons avec quelle préposition (14'-16') ils auraient pu être produits dans le contexte où ils se trouvent :

(14) we had pizza take-away [SR16]

(14') we had pizza [[to]] take-away

(15) it's like a ... outside things like tennis bats and badmington rackets [EB4]

(15') it's like a ...[[for]] outside things like tennis bats and badmington rackets

(16) it's like a game Cluedo [EB14]

(16') it's like a game [[of]] Cluedo

29 En français, de [də] est souvent réduit en d' [d]. L'élision du schwa par les entendants se traduit par une réalisation non syllabique, ce qui pourrait expliquer certains cas d'absence de la préposition dans nos corpus, tels que :

(17) la chambre mon frère (...) il y a un petit lit et un banc [SM10]

(17') la chambre [[de]] mon frère (...) il y a un petit lit et un banc

(18) c'est le bowling Montpellier [SM34]

(18') c'est le bowling [[de]] Montpellier

(19) le bébé a fini manger [JD237]

(19') le bébé a fini [[de]] manger

Toutefois, si cette hypothèse était valable dans tous les cas, nous ne devrions pas relever de cas où la préposition absente aurait été une préposition bien audible :

(20) après plus tard deux jours on est allés au Vieux Montréal [KG207]

(20') après plus tard [[pendant]] deux jours on est allés au Vieux Montréal

(21) puis il joue une poupée avec les cheveux jaunes [MJL67]

(21') puis il joue [[avec]] une poupée avec les cheveux jaunes

(22) ils rentrent leur lit [MR41]

(22') ils rentrent [[dans]] leur lit

(23) and we didn't get to sleep about one o'clock in the morning [LS15] 
(23') and we didn't get to sleep [[until]] about one o'clock in the morning corpus de 2006, nous ont donc amenée à faire une analyse sémantique des relations nonmarquées par une préposition dans nos corpus, afin de déterminer si certaines relations étaient prédominantes et pouvaient être rattachées à un développement cognitif propre aux sourds.

\subsection{Hypothèse cognitive}

C'est donc une hypothèse cognitive que nous avons voulu tester, par une analyse sémantique des énoncés dans lesquels la préposition est "manquante". Pour ce faire, nous avons construit une liste de valeurs sémantiques auxquelles les prépositions peuvent référer. A partir de l'index des notions présenté par Cadiot (1997: 279), nous avons adopté la typologie suivante: attribution/datif, but, cause, catégorisation (qualité et type), contenant, contenu, direction, localisation (spatiale et temporelle), métaphore, métonymie, possessif, quantité.

Nous avons procédé au classement des énoncés selon cette typologie pour nous intéresser en particulier aux valeurs sémantiques susceptibles d'être directement associées aux représentations cognitives pouvant être liées à l'audition : l'espace et le temps.

Dans les relations non-marquées par une préposition (Tableau 3), dominent les relations de localisation spatiale et temporelle, ainsi que les relations de catégorisation, qui, ensemble, représentent plus de $75 \%$ des cas : localisation spatio-temporelle : 37,6\% (spatiale : $32 \%$; temporelle : 5,6\%) ; catégorisation : $40 \%$ (qualité : $8,3 \%$; type : $31,6 \%$ ); autres : $22,5 \%$.

Tableau 3 : Types de relations non marquées par une préposition

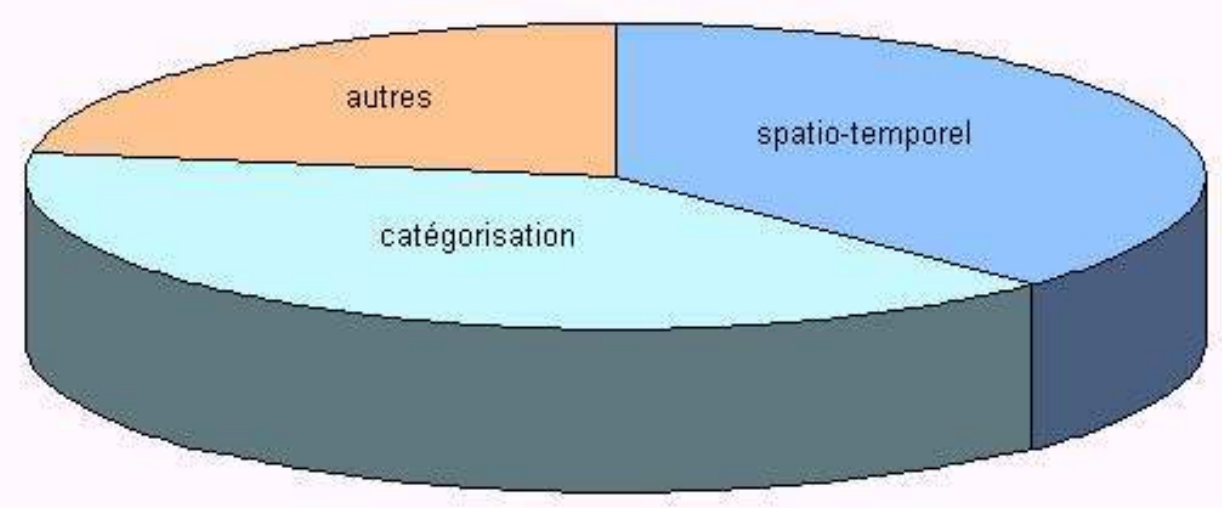

Les catégories de localisation et de catégorisation ont-elles des points communs au plan cognitif ? Ce pourrait être le cas, du fait que la catégorisation peut être représentée par 
des référents faisant appel à la localisation, qu'elle soit spatiale ou temporelle. En relation avec la catégorisation, on peut en effet évoquer :

- la localisation spatiale, puisque la hiérarchie type / sous-type est souvent représentée par une image d'emboîtement;

- la localisation temporelle, puisque le déroulement linéaire du temps peut permettre à la parole de donner de plus en plus de précisions, et donc de sous-catégoriser; par exemple, à partir de "avoir envie", il est possible d'indiquer par un supplément de parole qu'il s'agit de la sous-catégorie "avoir envie de jouer". L'apport de la précision par une postmodification s'inscrit dans le déroulement du temps. Or il est établi (Lafon, 1985) qu'une des façons de construire la représentation du temps se fait par la superposition du déroulement de la parole au déroulement du temps.

Catégorisation et localisation spatio-temporelle pourraient donc être reliées au plan cognitif. Les relations que les sujets ne marquent pas par une préposition seraient ainsi majoritairement rattachées à l'espace et au temps, dont nous avons vu que la perception est perturbée par la surdité profonde.

Voici quelques exemples d'énoncés problématiques du fait de l'absence de préposition, exemples présentés de façon à mettre en évidence des parallèles entre l'anglais et le français. L'existence de phénomènes identiques dans les deux langues pourrait indiquer le rôle de la surdité à cet égard.

Les énoncés (24) à (27) concernent des cas de localisation spatiale :
(24) droite [MR2]
(24') [[à]] droite
(25) it is left side [SB8]
$\left(25^{\prime}\right)$ it is [[on the]] left side
(26) ils rentrent leur lit [MR41]
(26) ils rentrent [[dans]] leur lit
(27) and the garage [MS23]
$\left(27^{\prime}\right)$ and [[in]] the garage

Les énoncés (28) à (31) concernent des cas de catégorisation :

(28) we had pizza take-away [SR16]

$\left(28^{\prime}\right)$ we had pizza [[to]] take-away

(29) un auto transport [KG115]

(29') un auto [[de]] transport

(30) it's like a game Cluedo [EB14]

(30') it's like a game [[of]] Cluedo

(31) c'est le bowling Montpellier [SM34]

(31') c'est le bowling [[de]] Montpellier

L'hypothèse cognitive peut être étayée par la mise en relation de nos observations avec ce qui se passe dans les langues des signes. En effet, les langues signées ont été créées par les sourds eux-mêmes, dès qu'ils se trouvaient entre eux, y compris lorsqu'ils étaient regroupés dans des institutions destinées à leur enseigner la langue orale. Nous pouvons faire l'hypothèse qu'elles sont la trace de conceptions cognitives propres aux sourds.

L'article de de Vriendt et Rasquinet (1990) décrit avec précision les prépositions dans la Langue des Signes Belge (LSB). Sont identifiées d'une part les relations qui font l'objet d'un signe à valeur de préposition, d'autre part les relations qui ne sont pas signées.

Les auteurs établissent la typologie suivante :

- prépositions de nature dimensionnelle :

a. situationnelle (pas de signe) ; 
b. relationnelle (pas de signe s'il s'agit d'espace; signe possible mais non systématique s'il s'agit de temps);

c. destination ou origine du mouvement (pas de signe) ;

d. durée (pas de signe s'il s'agit d'unités de temps, par exemple "3 mois" ; signe s'il s'agit d'une période, par exemple "l'été").

- prépositions de nature non dimensionnelle :

a. cause (pas de signe) ;

b. but (pas de signe) ;

c. moyen (signe possible mais non systématique).

- prépositions aux emplois figés :

a. complément de nom (pas de signe, sauf si le nom réfère à un humain) ;

b. complément du verbe (pas de signe);

c. complément de l'adjectif (pas de signe).

La typologie de de Vriendt et Rasquinet est différente de celle que nous avons utilisée dans notre propre classement. Il est toutefois possible d'établir des correspondances. Les localisations temporelles et spatiales de notre étude correspondent aux différents cas de prépositions de nature dimensionnelle; les relations de catégorisation de notre étude correspondent aux emplois figés, aux compléments de nom et compléments d'adjectif. problématiques. Toutefois, nous limiterons la discussion aux trois thèmes suivants : la prédication et les groupes syntaxiques; la spécificité de la préposition "pour"; la représentation visuelle des relations entre référents.

\subsection{Prédication et groupes syntaxiques}

Les observations du paragraphe 3 montrent que les sourds manipulent la prédication de telle sorte que sa cohésion puisse en être affectée. Ce résultat peut être mis en relation avec l'appréhension spécifique des groupes syntaxiques par les sourds telle que l'a révélée l'analyse des reprises et des répétitions dans le corpus de 1988 (Vincent-Durroux, 1992). 
Cette analyse montre que, contrairement aux entendants qui "remontent" au début du syntagme en cas de reprise ou d'auto-correction ou de répétition (Blanche-Benveniste, 1985), prenant en compte l'intégrité du syntagme, ce n'est pas le cas des sourds. Par exemple, l'énoncé produit par un sourd "Là c'était ma chambre avec les greniers $\varnothing$ des greniers" pourrait prendre la forme suivante chez un entendant : "Là c'était ma chambre avec les greniers avec des greniers".

Les énoncés cités dans le paragraphe 3 peuvent concerner la production de précisions (énoncés 3 à 5). Il s'agit donc d'auto-corrections, qui devraient exiger la prise en compte des groupes syntaxiques et pourraient conduire à des répétitions de structures syntagmatiques, qui sont justement évitées dans ces énoncés. A titre d'illustration, dans l'énoncé (8), la structure de groupe prépositionnel devrait être répétée, avec correction de la préposition, comme en $\left(8^{\prime}\right)$ :

$\left(8^{\prime}\right)$ and it was very hot because it is in June on June $19^{\text {th }}$

\subsection{Spécificité de la préposition "pour"}

51 L'emploi de "pour" est fautif dans deux énoncés que nous avons commentés; dans le contexte de l'énoncé (5), un entendant utiliserait probablement "avec" (je partage la dépense avec mes parents) et dans le contexte de l'énoncé (10), "quant à" pourrait convenir (quant à mon dernier anniversaire, je l'ai passé à Colmar).

Cette observation est cohérente avec le rôle particulier de "pour" au sein de l'ensemble des prépositions. Dans le développement du langage chez l'enfant, il semble que la richesse phonologique et lexicale de cette préposition la rende apte à des emplois synthétiques, tels que "le doudou pour Madeleine", interprétables comme: "le doudou pour que Madeleine console Maman" (Morgenstern et Sekali, 2007). La préposition "pour" aurait aussi un rôle de "joker" chez les sujets souffrant d'aphasie grammaticale (Sahraoui, 2007) pour remplacer les prépositions non disponibles chez le sujet; on peut faire l'hypothèse que cela se produit ainsi chez les aphasiques parce que cette préposition a été associée à une multiplicité de valeurs et a contribué à structurer la catégorie des prépositions. Dans les énoncés (5) et (10), ce rôle de "joker" ou d'opérateur polyvalent pourrait aussi être envisagé : "pour" marquerait l'établissement d'une relation, sans que la relation ne soit définie davantage.

\subsection{La représentation visuelle des relations entre référents}

Il semble y avoir des liens importants entre l'absence de préposition et la représentation des relations spatiales et temporelles entre des référents ( $c f$. paragraphe 4 ci-dessus), et ce, quelle que soit la modalité d'expression (langue orale chez des oralistes, langue signée) : les sourds ne marquent pas les relations spatio-temporelles aussi souvent que le font les entendants. En langues signées, cela peut s'expliquer par le fait que les signes occupent un espace qui peut demeurer dans la mémoire visuelle. S'il faut établir des relations entre les référents de ces signes, la contiguïté des signes peut suffire. Il semblerait que les sourds oralistes adoptent une stratégie identique, en s'appuyant sur une représentation visuelle des référents plutôt que sur une représention auditive, bloquée par la surdité. 


\section{Conclusion} différentes populations ayant pour point commun la surdité profonde. Ils permettent d'indiquer que la perturbation des perceptions spatio-temporelles peut conduire à une perturbation dans la représentation des relations de localisation et de catégorisation, ce qui, dans la langue, se manifeste en particulier dans l'emploi des prépositions.

Ces résultats contribuent à préciser certains aspects du langage chez les sourds ; malgré les gains auditifs procurés par les implants cochléaires chez nos sujets, il ressort que des spécificités affectant la relation prédicative, l'espace et le temps, demeurent. Les connaître pourrait permettre aux thérapeutes qui accompagnent le développement linguistique des jeunes sourds d'en tenir compte pour orienter leur prise en charge de façon plus spécifique.

\section{BIBLIOGRAPHIE}

Blanche-Benveniste C. (1985). "La dénomination dans le français parlé : une interprétation pour les répétitions et les hésitations". Recherches sur le français parlé. vol. 6 : pp. 109-130. Aix-enProvence : Groupe Aixois sur le Français Parlé.

Bochner J.H. \& Albertini J.A. (1988). "Language varieties in the deaf population and their acquisition by deaf children and adults". In Strong, M. (éd.). Language Learning and Deafness. Cambridge : Cambridge University Press. pp. 3-48.

Cadiot P. (1997). Les prépositions abstraites en français. Paris : Armand Colin.

Charrow V.R. (1974). "Deaf English : An investigation of the written English competence of deaf adolescents". Stanford University, Psychology and Education Series, Institute for Mathematical Studies in the Social Sciences, Technical Report, $\mathrm{n}^{\circ} 236$.

Franckel J.J. (2007). "Prépositions et rection verbale". Communication au colloque international "Autour de la préposition", CRISCO, CNRS, Université de Caen.

Franckel J.J. \& Paillard D. (2007). Grammaire des prépositions. Tome I. Paris : Ophrys.

Groussier M.-L. (1997). "Préposition et primarité du spatial". Faits de langue. La préposition : une catégorie accessoire ? vol. 9 : pp. 221-234. Paris : Ophrys.

Geers A.E., Nicholas J., Tye-Murray N., Uchanski R., Brenner C., Davidson L., Toretta G., \& Tobey E. (2000). "Effects of communication mode on skills of long-term cochlear implant users". Annals of Otology, Rhinology and Laryngology. vol. 109, nº 12 (2), supp. 185, December : pp. 89-92.

Huart R. (2002). Grammaire orale de l'anglais. Paris : Ophrys.

Ivemey G. \& Lachterman D. (1980). "The written language of young English deaf children". Language and Speech. $\mathrm{N}^{\circ} 23:$ pp. 351-377.

Jimenez M. (1997). La psychologie de la perception. Paris : Flammarion collection Dominos. 
Lafon J.-C. (1985). Les enfants déficients auditifs. Villeurbanne : SIMEP.

Miyamoto R.T., Kirk K.I., Svirsky M. A. \& Sehgal S. T. (1999). "Communication skills in cochlear implant recipients". Acta Oto-Laryngologica. vol. 119, n 2 : pp. 219-224.

Morgenstern A. \& Sekali M. (2007). "La naissance d'une catégorie : étude contrastive de l'émergence des prépositions chez l'enfant en anglais et en français". Communication au colloque international "Autour de la préposition", CRISCO, CNRS, Université de Caen.

Lacerte L. (1988). "La langue des signes québécoise (LSQ) et le français : difficultés à l'écrit chez la personne sourde". Mémoire de maîtrise sous la direction du Professeur C. Dubuisson. Université du Québec à Montréal.

O’Donoghue G.M. (1999). "Hearing without ears : do cochlear implants work in children ?". British Medical Journal, $n^{\circ} 318:$ pp. 72-73.

Ouellet C., Le Normand M.-T. \& Cohen H. (2001). "Language evolution in children with multichannel cochlear implants". Brain and Cognition, vol. 46, n 1-2, June-July : pp. 231-235.

Sahraoui H. (2007). "Une étude des prépositions à travers l'aphasie agrammatique". Communication au colloque international "Autour de la préposition", CRISCO, CNRS, Université de Caen.

Vieu A., Mondain M., Blanchard K., Sillon M., Reuillard-Artières F., Tobey E., Uziel A.\& Piron J. P. (1998). "Influence of communication mode on speech intelligibility and syntactic structure of sentences in profoundly hearing impaired French children implanted between five and nine years of age". International Journal of Pediatric Otorhinolaryngology. $\mathrm{N}^{\circ} 44:$ pp. 15-22.

de Vriendt S. \& Rasquinet M. (1990). "Prepositions in a Belgian Sign Language". In : Edmondson, W.H. et F. Karlsson (éds). SLR '87. Papers from the Fourth International Symposium on Sign Language Research. Hambourg : Signum Verlag. pp. 57-65.

Vincent-Durroux L. (1990). "Problèmes d'acquisition du langage par les adolescents déficients auditifs profonds oralistes, en français et en anglais". Thèse de Doctorat ès Lettres (Ph.D.), sous la direction des Professeurs J.-M. Léard et C. Dubuisson. Université de Sherbrooke (Canada). 286 pages.

Vincent-Durroux L. (1992). "La langue orale des sourds profonds oralistes". Thèse de doctorat de linguistique générale, sous la direction du Professeur A. Joly. Université Paris 4. 374 pages.

\section{NOTES}

1. Une version préalable à cet article a été présentée au colloque "Autour de la préposition" (CRISCO - CNRS, Caen, 2007) sous le titre "Observations liées à l'emploi des prépositions chez des enfants et adolescents sourds profonds, en anglais et en français".

2. Nous tenons à remercier les enfants et adolescents qui ont pris part à cette étude, avec l'autorisation de leurs parents. Nous remercions également les orthophonistes, directeurs d'instituts ou membres d'associations de parents, pour leur accueil et leurs conseils. Enfin, nous remercions les participants au colloque "Autour de la préposition" (CRISCO - CNRS, Caen, 2007) dont les communications et les commentaires nous ont permis d'affiner l'étude des prépositions dans nos corpus.

3. Suite au diagnostic d'une surdité profonde bilatérale chez leur enfant, les parents doivent rapidement choisir un mode de communication : il peut s'agir de la langue signée dans leur pays ; de la langue orale de leur communauté (oralisme); du langage parlé complété (LPC) qui associe 
parole et indications manuelles destinées à lever certaines ambiguïtés en lecture labiale (ex. "pain" / "bain"), ou du bilinguisme (langue signée et langue orale).

\section{RÉSUMÉS}

La surdité profonde prélinguistique peut avoir des conséquences sur la représentation du temps et de l'espace, lesquelles peuvent se manifester dans les productions linguistiques des sourds. Cet article est consacré à l'analyse de l'emploi des prépositions dans un corpus oral recueilli auprès de sujets oralistes anglophones ou francophones, en vue d'hypothèses sur les spécificités du langage des sourds profonds. Nous traitons les énoncés problématiques selon que la préposition y est inadaptée, superflue ou manquante. Dans les deux premiers cas, l'analyse fait apparaître des problèmes de linéarité syntaxique. En cas de préposition manquante, après une hypothèse phonologique, nous formulons une hypothèse cognitive, en relation avec l'espace, le temps, la catégorisation et la description des prépositions en Langue des Signes Belge. La dicussion porte sur la prédication, la spécificité de la préposition "pour" et le rôle possible de la représentation visuelle des relations entre référents.

Prelingual profound deafness may have consequences on the representation of space and time, consequences which may appear in the deaf's linguistic productions. This paper presents the analysis of the use of prepositions in oral data gathered from oral deaf subjects who speak either French or English; the analysis aims at hypotheses on specificities in the language of the profoundly deaf. Problematic utterances are related to superfluous, inadequate or missing prepositions. In the first two cases, there are problems in syntactic linearity. As concerns missing prepositions, phonological and cognitive hypotheses can be considered, in relation to space, time, categorisation, and the description of prepositions in Belgian Sign Language. The results are discussed in terms of predication, of the specificity of the French preposition "pour", and the possible role of visual representation of the relationships between referents.

\section{INDEX}

Mots-clés : représentation, temps, espace, surdité profonde, anglais, français, préposition

Keywords : representation, time, space, profound deafness, English, French, preposition

\section{AUTEUR}

\section{LAURENCE VINCENT-DURROUX}

Université Montpellier 3, Centre d'Etude de Linguistique Anglaise (CELA), EA 741 